\title{
COMUNICAÇÃO ZIGBEE APLICADA EM UM SISTEMA DE CONTROLE
}

\author{
F. D. FAGUNDES ${ }^{1 *}$, L. P. FAGUNDES ${ }^{1}$, M. J. CUNHA ${ }^{2}$ e F. V. R. SILVA ${ }^{2}$ \\ ${ }^{1}$ Centro Federal de Educação Tecnológica de Minas Gerais \\ ${ }^{2}$ Universidade Federal de Uberlândia \\ fredericofagundes.cefetmg@gmail.com*
}

Artigo submetido em agosto/2015 e aceito em janeiro/2016

DOI: $10.15628 /$ holos.2016.3786

\section{RESUMO}

O presente artigo apresenta o desenvolvimento de uma interface de comunicação entre os padrões EIA RS-232 e ZigBee. Sistemas sem fio não apresentam custos extras com fiação e infraestrutura, permitem fácil realocação e a adição de novos dispositivos é simples. O padrão RS-232 é utilizado em diversas a plicações e o objetivo do projeto foi agregar as vantagens do padrão de comunicação sem fio ZigBee em um sistema que utilize o RS-232. O projeto foi aplicado em sistemas didáticos de controle de processos, no qual foi desenvolvida uma placa com um módulo ZigBee e um sistema de conversão do sinal serial RS-232. Os testes mostraram que a interface não interferiu na infraestrutura do sistema existente, permitiu que a estação de trabalho fosse realocada e ainda permitiu que uma única estação se comunicasse com mais de um sistema.

PALAVRAS-CHAVE: Comunicação sem fio, Controle de processos, Conversão de dados.

\section{ZIGBEE COMMUNICATION APPLIED IN A CONTROL SYSTEM}

\begin{abstract}
This paper presents the development of a communication interface between the standards EIA RS232 and ZigBee. Wireless systems do not have extra costs of wiring and infrastructure, allow easy relocation and addition of new devices is simple. The RS-232 is used in various applications and the project's goal was to add the standard advantages of ZigBee wireless communication in a system previously using the RS-232. The project was
\end{abstract}

\begin{abstract}
implemented in educational process control systems. A board was developed, containing a ZigBee module and a conversion system for the RS-232 serial signal. The tests showed that the interface does not interfere with the existing system infrastructure, allowed the workstation to be reallocated and still allowed a single station to communicate with more than one system.
\end{abstract}

KEYWORDS: Wireless communication, Process Control, Data conversion. 


\section{INTRODUÇÃO}

Na indústria, predomina a utilização de cabeamento para a comunicação dos instrumentos de campo com os controladores e sistemas de supervisão. São inúmeros cabos saindo das salas de controle e supervisão para o chão-de-fábrica, recebendo e transmitindo os mais diversos tipos de dados (RAMOS, 2012).

A principal vantagem do uso de cabos é a confiabilidade na transmissão de dados, ou seja, há poucas perdas nos dados que precisam ser transmitidos e recebidos de um ponto a outro. Outro fator importante é que os instrumentos instalados no campo necessitam de cabeamento para alimentação e, muitas vezes, é viável que a transmissão de dados seja feita através da mesma estrutura e, em alguns casos, até pelo mesmo cabo (ALVES, 2010).

Um padrão bastante utilizado em sistemas cabeados, desenvolvido pela EIA (Electronic Industries Alliance), é o RS-232. Consiste em um padrão de comunicação digital serial, utilizado tanto em portas seriais de computadores quanto em conjunto com protocolos de comunicação industrial (ALBUQUERQUE; ALEXANDRIA, 2009).

Com o desenvolvimento no setor de transmissão de dados, surgem novas tecnologias, abrindo mais opções para as empresas. Uma das tecnologias que mais se desenvolveu é a transmissão sem fio (wireless), em que o transmissor e o receptor comunicam-se sem a necessidade de cabeamento (RAMOS, 2012).

Esse tipo de transmissão já é muito utilizado e substitui bem a comunicação por cabeamento em alguns casos, como na transmissão de voz por radiofrequência, na telefonia e na internet.

Inicialmente, não se utilizava essa tecnologia na indústria devido à perda relativamente alta de dados em sua transmissão, causada por interferências de campos magnéticos e barreiras físicas, por exemplo (RAMOS, 2012).

As redes industriais que utilizam transmissão por cabeamento necessitam de altos investimentos em infraestrutura e mão-de-obra, sempre que a rede industrial sofre manutenção ou é expandida. Já com a utilização de redes sem fio há redução do custo da infraestrutura e simplificação das instalações, pois não há necessidade de uso de bandejas e dutos. O custo de manutenção também é reduzido, pois, sem o cabeamento, a substituição de dispositivos é facilitada (RAMOS, 2012).

Atualmente, há opções de tecnologias de transmissão sem fio que prometem baixa interferência, alta confiabilidade na transmissão de dados e trabalham com baixa potência. Uma dessas tecnologias é o padrão ZigBee, que vem sendo utilizado com sucesso no processamento de sinais biomédicos (ANDRIGHETTO, 2008), no monitoramento de parques eólicos (PEREZ; HELENA, 2011) e até no sensoriamento remoto de pranchas de surfe (BONA; FERREIRA; SCHWARZ, 2012).

Entretanto, essas vantagens e aplicações consideram a implantação do padrão ZigBee desde o início do projeto. Em sistemas cuja estrutura já está cabeada, essa implantação pode não ser atrativa. Pensando nisso, o projeto visou agregar vantagens a um sistema já cabeado, mas que apresentava, principalmente, duas desvantagens. Primeira, se houver a intenção de expandir um sistema cabeado, o custo de infraestrutura é alto. Ademais, a mobilidade dos dispositivos é, no mínimo, trabalhosa. 
Devido à ampla utilização do RS-232 (ou EIA232), foi pensado que uma interface entre este padrão e o padrão de comunicação sem fio ZigBee, demonstraria que um sistema cabeado pode apresentar as vantagens da transmissão de dados wireless sem que sua infraestrutura sofresse modificações.

O objetivo foi desenvolver uma placa de comunicação que servisse como interface entre os padrões RS-232 e ZigBee. Além disso, visou-se aplica-la em um sistema que utiliza o padrão RS232, para avaliar sua efetividade.

Um dos sistemas onde a placa foi aplicada é uma planta didática de controle de temperatura CRT (Controllo e Regolazione Temperatura), da marca Didacta ${ }^{\circledR}$, localizada no Laboratório de Automação e Controle do CEFET-MG, em Araxá. Seu esquema de funcionamento é ilustrado na Figura 1.

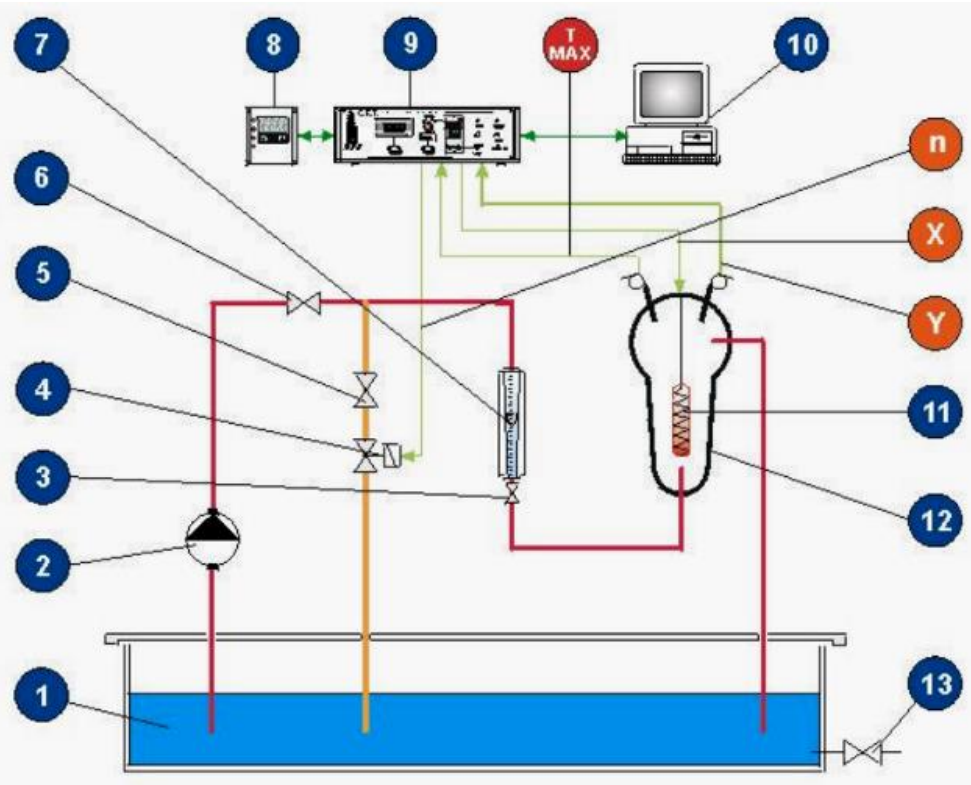

Figura 1: Esquema da planta didática CRT.

Fonte: Didacta Italia, 2012.

O sistema conta com um reservatório de água (1), uma bomba (2), uma válvula de controle de alimentação (3), uma válvula de simulação de distúrbio (4) (sinal representado por n) e uma resistência para aquecimento da água (11). A água do reservatório é bombeada para um bulbo (12), onde se encontram a resistência responsável pelo aquecimento da água (alimentação da resistência representada por $\mathrm{X}$ ) e transmissores de temperatura (sinal representado por $\mathrm{Y}$ ). A válvula de controle (3) tem sua vazão monitorada por um indicador (7). As válvulas (5) e (6) são para controle manual de abertura ou fechamento de suas respectivas tubulações. Há uma válvula para esgotamento do tanque (13), se necessário. Os equipamentos (8) e (9) representam a interface da planta, podendo também ser utilizados para controle eletrônico sem uso do computador (10). O computador (10) é uma estação de trabalho que recebe os dados da planta através de interface serial RS-232 e conta com um software próprio para controle, disponibilizado pelo fabricante (DIDACTA ITALIA, 2012).

Na seção 2 do trabalho, é descrita a metodologia de implantação do projeto e os testes realizados. Na seção 3, os resultados dos testes são apresentados, bem como as inferências 
alcançadas com os resultados. A seção 4 apresenta as conclusões obtidas. As publicações e demais referências consultadas para o desenvolvimento desse trabalho são listadas na seção 5 .

\section{METODOLOGIA}

O ZigBee é um padrão de comunicação aberto, por isso existem diversos fabricantes e dispositivos para realizar comunicação com esse padrão. Um desses fabricantes é a Fractum ${ }^{\circledR}$, responsável pelo desenvolvimento do $U_{B e e M a x}{ }^{\circledR}$. O dispositivo possui uma boa relação custo/benefício comparado com os módulos ZigBee de outros fabricantes, por isso foram escolhidos para a realização dos testes práticos.

Os módulos UBeeMax ${ }^{\circledR}$ contam com uma porta de comunicação serial assíncrona, que pode comunicar com outros dispositivos compatíveis com os níveis lógicos e de tensão do padrão USART (Universal Synchronous Asynchronous Receiver Transmitter). O USART é um padrão de comunicação serial, usado como referência em dispositivos e padrões que utilizam tanto comunicação síncrona quanto assíncrona.

As plantas industriais do Laboratório de Automação e Controle enviam os dados para a estação de trabalho através de cabo par trançado, com base no padrão RS-232. O protocolo RS232 pode tornar-se compatível com o padrão USART por meio da conversão do nível de tensão. Os módulos UBeeMax ${ }^{\circledR}$ utilizam níveis de tensão entre 2,4 e 3,6 Volts para seus bits, já o padrão RS232 comunica-se utilizando níveis entre 3 e 25 Volts, positivos ou negativos.

Para que o módulo pudesse estabelecer a comunicação com o padrão RS-232 da planta, foi, então, utilizado o Circuito Integrado $(\mathrm{Cl}){\mathrm{Max} 3232^{\circledR}}^{\circledR}$, da MaxStream ${ }^{\circledR} . \mathrm{O} \mathrm{Cl}$ tem a função de baixar os níveis de tensão do padrão RS-232, para que se tornem compatíveis com a porta USART do módulo ZigBee, e aumentar os níveis de tensão da porta USART para conciliar com o padrão RS232. Foram utilizados capacitores associados ao $\mathrm{Cl}$ Max3232 ${ }^{\circledR}$, conforme indicação do fabricante (MAXIM INTEGRATED, 2007). A Figura 2 mostra o esquema de ligação do Max3232 ${ }^{\circledR}$.

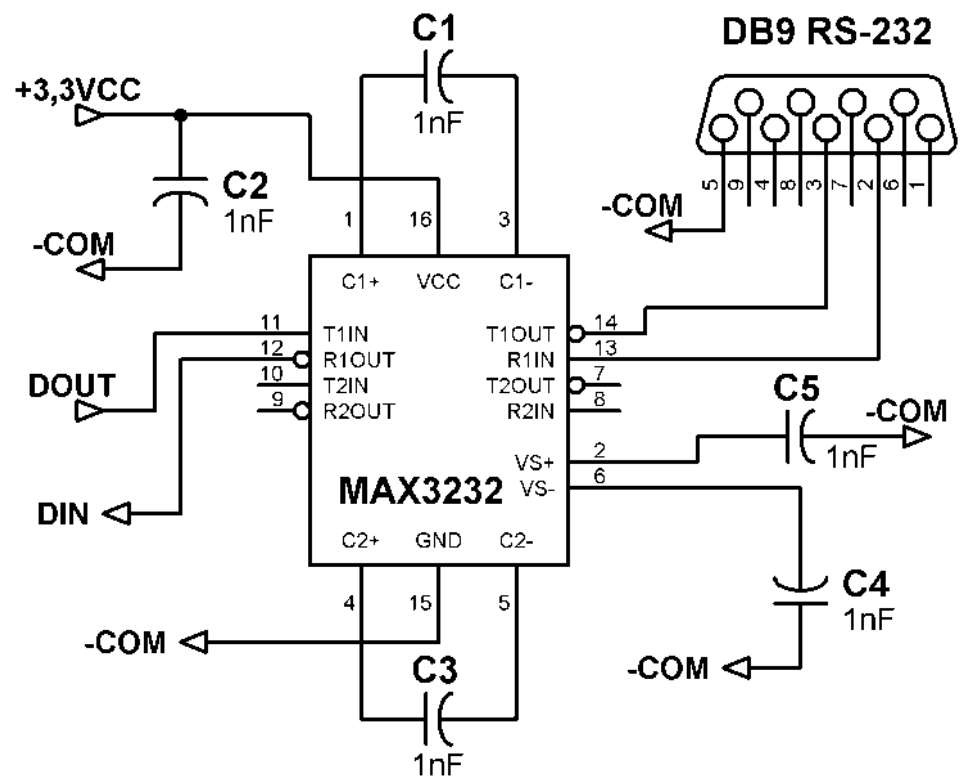

Figura 2: Diagrama de ligação do $\operatorname{Max} 3232^{\circledR}{ }^{\circledR}$ com a porta serial RS-232. 
Os terminais 11 (T1IN) e 12 (R1OUT) do $\mathrm{Cl}$ MAX3232 ${ }^{\circledR}$ estão em comunicação com os pinos 2 e 3, respectivamente, do módulo ZigBee. A ligação do módulo ZigBee pode ser vista na Fig ura 3.

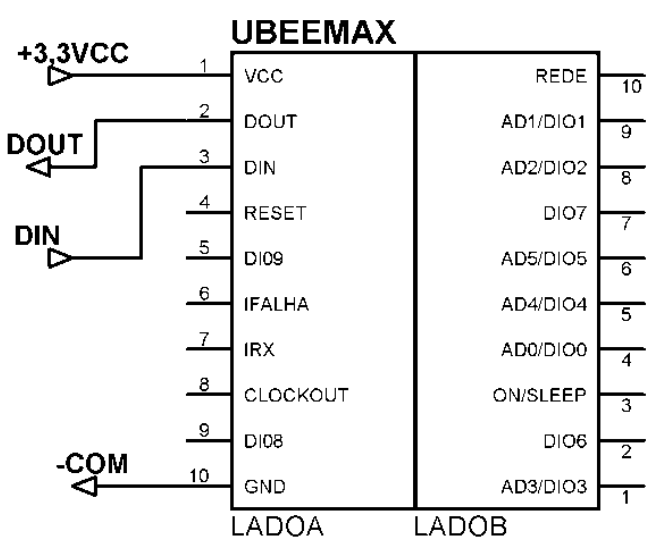

Figura 3: Diagrama de ligação do módulo UBeeMax ${ }^{\circledR}$.

Ambos Cls necessitam de tensão de alimentação de 3,3 Volts, como pode ser visto nas Figuras 2 e 3. A placa foi desenvolvida de modo que pudesse receber diversos níveis de tensão. Para isso, foi utilizado o regulador de tensão ajustável LM317 ${ }^{\circledR}$, da Texas Instruments ${ }^{\circledR}$, capaz de $^{2}$ receber até 37 Volts na entrada. A tensão de saída é ajustada de acordo com a associação dos resistores R1 e R2, mostrados na Figura 4 (TEXAS INSTRUMENTS, 2014).

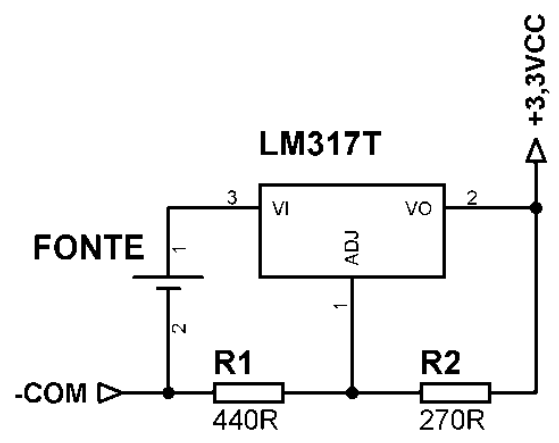

Figura 4: Diagrama de ligação do $\mathrm{LM} 317^{\circledR}$.

Seguindo o projeto, a placa foi desenvolvida e montada na saída da interface eletrônica da planta didática. A placa final, com a conexão RS-232, é mostrada na Figura 5.

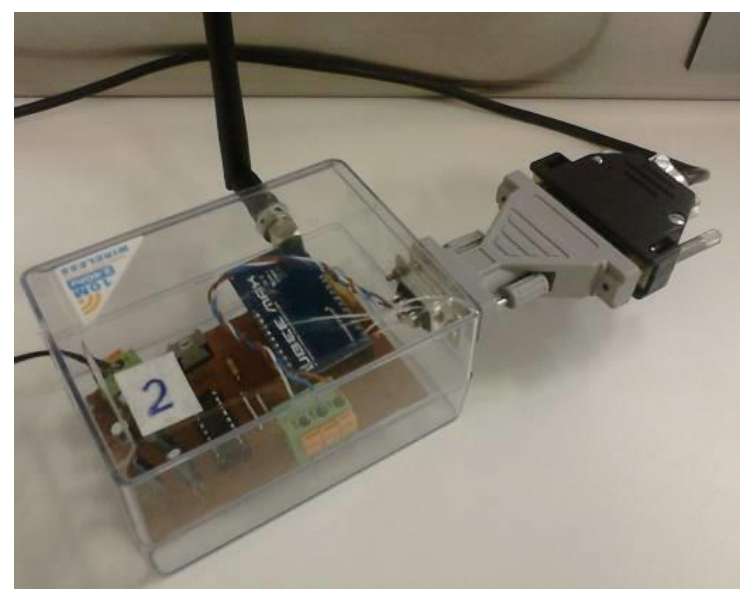

Figura 5: Placa de comunicação finalizada conectada à porta serial RS-232 da planta. 
Para se comunicar com a planta, a estação de trabalho conta com uma interface mais simples de trabalhar com ZigBee, que é a USB (Universal Serial Bus). O fabricante Fractum ${ }^{\circledast}$ fornece o dispositivo USBee ${ }^{\circledR}$ para que o módulo UBeeMax ${ }^{\circledR}$ possa ser conectado à porta USB da estação. O módulo USBee ${ }^{\circledR}$ associado ao UBeeMax ${ }^{\circledR}$ é mostrado na Figura 6.

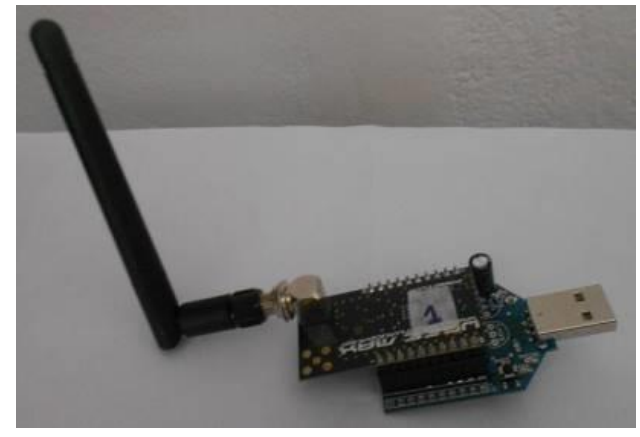

Figura 6: Módulo USBee ${ }^{\circledR}$ associado ao UBeeMax ${ }^{\circledR}$.

Com a placa de comunicação na planta e o módulo na estação de trabalho, foi iniciada a troca de dados através do padrão ZigBee. O teste inicial foi de mobilidade, utilizando um notebook como estação de trabalho e afastando-o da planta até que o sistema apresentasse perda de dados na comunicação. Para avaliar a perda de dados, foram quantificados os bits enviados e recebidos na comunicação e, quando houve perda ou alteração de valores nos bits, considerou-se falha na comunicação.

O segundo teste avaliou a expansibilidade da rede. Foram desenvolvidas outras duas placas iguais e as mesmas foram aplicadas a outras duas plantas didáticas de controle, do mesmo fabricante. Foram necessárias alterações no software responsável pelo envio de requisições e recebimento das respostas. Então, foi avaliada a possibilidade de comunicação com todas as três plantas.

\section{RESULTADOS E DISCUSSÕES}

O primeiro teste, de mobilidade, mostrou que a comunicação entre os dispositivos começou a falhar quando a distância entre os mesmos chegou a aproximadamente 80 metros. Isso em uma situação onde a estação de trabalho estava em ambiente aberto e a planta didática no laboratório.

Quando a estação de trabalho passou para um ambiente fechado, aumentando assim os obstáculos físicos entre os dispositivos, a perda passou a ocorrer em uma distância de aproximadamente 40 metros. Esse teste foi feito mantendo a planta no laboratório e movendo a estação de trabalho para outro laboratório. A imagem de satélite da Figura 7 representa essas duas etapas.

O fabricante especifica que o módulo UBeeMax ${ }^{\circledR}$ alcança 300 metros em ambientes fechados (FRACTUM, 2015), entretanto, supõe-se que esse valor seja relativo a interrupção total na comunicação, não a ocorrência de falhas na transmissão. Para esse trabalho, a distância alcançada foi considerada aceitável. Em aplicações que exijam longas distâncias, é possível utilizar módulos ZigBee como roteadores (MESSIAS, 2008). 


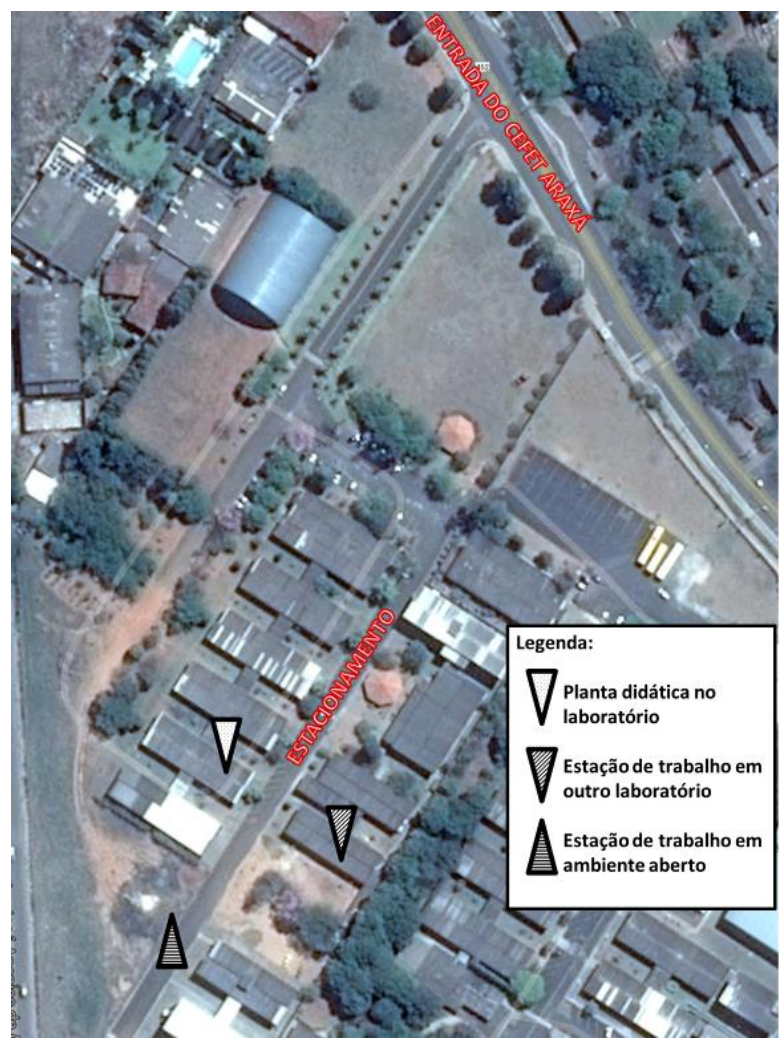

Figura 7: Local dos testes.

Em ambiente fechado, à distância de 40 metros, a transmissão apresentou falhas em $5,696 \%$ dos ciclos. Cada ciclo consiste de uma mensagem de requisição de 16 bits e uma mensagem de resposta de 16 bits. Considerando as perdas bit a bit, o percentual de falhas foi de 0,178\%. A Tabela 1 demonstra as medições realizadas durante alguns testes. A Tabela 2 mostra os resultados dos testes. Os testes em ambiente aberto apresentaram resultados similares.

Tabela 1: Falhas ocorridas na comunicação entre a planta e a estação de trabalho.

\begin{tabular}{c|c|c|c}
\hline $\begin{array}{c}\text { Ciclos por } \\
\text { teste }\end{array}$ & Falhas & $\begin{array}{c}\text { Bits } \\
\text { transmitidos }\end{array}$ & $\begin{array}{c}\text { Bits com } \\
\text { falha }\end{array}$ \\
\hline 35 & 1 & 1120 & 1 \\
1 & 1 & 32 & 1 \\
2 & 1 & 64 & 1 \\
6 & 1 & 192 & 1 \\
5 & 1 & 160 & 1 \\
25 & 1 & 800 & 1 \\
10 & 1 & 320 & 1 \\
11 & 1 & 352 & 1 \\
20 & 0 & 640 & 0 \\
20 & 0 & 640 & 0 \\
3 & 1 & 96 & 1 \\
\hline
\end{tabular}

Tabela 2: Resultados das medições de falhas.

\begin{tabular}{c|c}
\hline Resultado analisado & Valor \\
\hline Total de ciclos & 158 \\
Total de falhas & 9 \\
Total de bits transmitidos & 5056 \\
Total de bits com falha & 9 \\
Percentual de falhas por ciclo & $5,696 \%$ \\
Percentual de falhas por bit & $0,178 \%$ \\
\hline
\end{tabular}


As falhas ocorridas na transmissão poderiam ser contornadas com algum algoritmo de verificação de erros, como a verificação de paridade, o checksum e o Cyclic Redundancy Check. Para a aplicação dessas técnicas, é necessária a adição de bits, o que não é possível, visto que a transmissão segue um padrão próprio nessas plantas de controle.

O segundo teste realizado foi de expansibilidade. Com ele a estação de trabalho passou a comunicar com três plantas de controle. Um software desenvolvido para esse fim mostra e armazena os dados das três plantas. A Figura 8 mostra um gráfico obtido durante o teste.

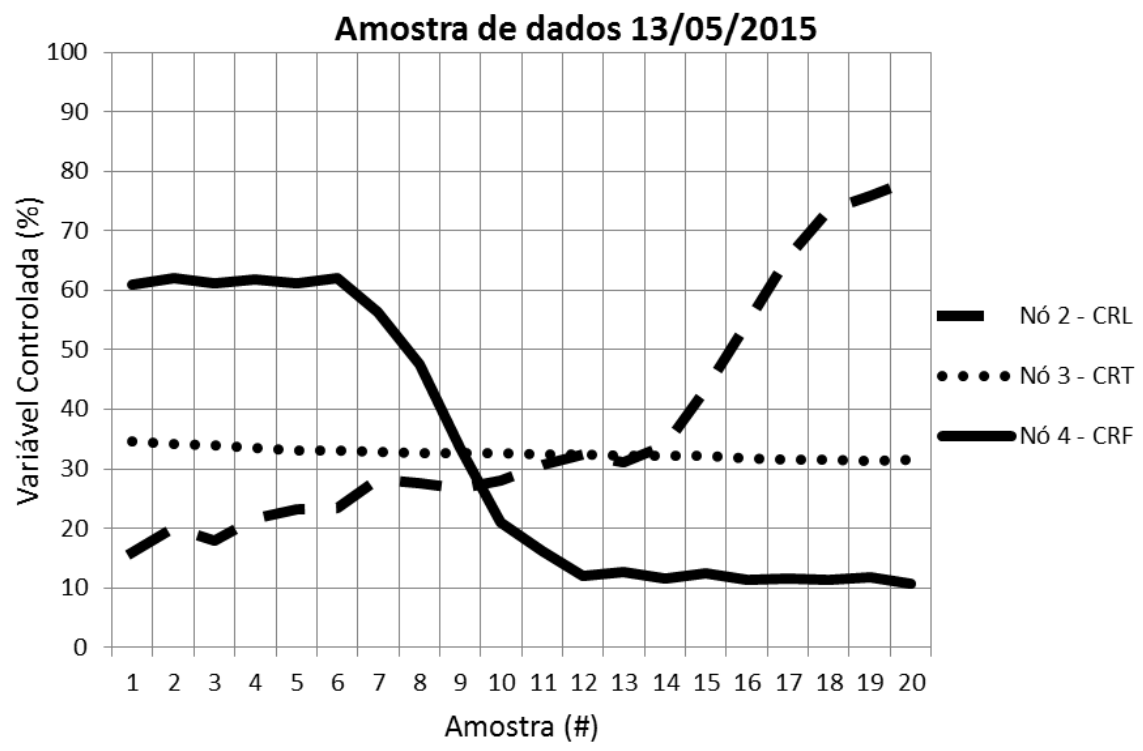

Figura 8: Amostra de dados.

A comunicação com as três plantas apresentou aumento de três vezes no tempo de varredura. Isso porque as plantas se comunicam com a estação de trabalho através de requisição e resposta, com isso para cada nova variável lida de uma nova planta de controle, o tempo para completar um ciclo de leitura aumenta proporcionalmente.

Com o monitoramento de três plantas de controle por uma estação de trabalho, foi demonstrada a expansibilidade do sistema. Essa expansão foi feita de forma simples, apenas com a adição de novas placas de comunicação e com alterações no software. Com essa aplicação, as plantas de controle, que são monovariáveis, podem ser consideradas como parte de um único sistema, possibilitando realizar estudos mais amplos envolvendo diversas variáveis.

\section{CONCLUSÃO}

O trabalho mostrou que é possível agregar vantagens de sistemas sem fio em sistemas cabeados. A implantação da interface possibilitou que a estação de trabalho fosse realocada, respeitando as limitações de distância, e que a rede fosse expandida sem dificuldades.

Além disso, a interface serial foi substituída pela USB. Isto eliminou uma limitação do sistema, que é o fato de notebooks geralmente não contarem com interfaces RS-232, ampliando as possibilidades de estações de trabalho.

Com a implantação da placa em diversas plantas de controle, também é possível desenvolver estudos em sistemas multivariáveis. Para verificação e correção dos erros na 
transmissão, evidencia-se a necessidade de desenvolver uma técnica que não necessite de adição de bits na transmissão.

\section{REFERÊNCIAS}

1. AlbuQUerque, P. U., \& AleXANDRIA, A. R. (2009). Redes Industriais (2 ed.). São Paulo: Ensino Profissional.

2. ALVES, J. L. (2010). Instrumentação, Controle e Automação de Processos (2 ed.). Rio de Janeiro: LTC.

3. ANDRIGHETTO, E. (2008). Sistema de Processamento de Sinais Biomédicos: Rede Wireless ZigBee com Aplicação do Padrão IEEE 802.15.4. Florianópolis, SC: UFSC.

4. BONA, D. D., FERREIRA, G. d., \& SCHWARZ, L. (2012). Sensoriamento Remoto em Pranchas de Surfe utilizando Tecnologia ZigBee. Florianópolis, SC: IFSC.

5. DIDACTA ITALIA. (2012). CRT - Temperature Control and Regulation Study Unit. Acesso em 12 de Maio de 2015, disponível em http://didacta.it/allegati/main_catalogs/CE_CRT_E.PDF

6. FRACTUM. (2015). Folha de dados Módulo RF U-BEEMAX. Acesso em 03 de Maio de 2015, disponível em http://www.fractumrf.com/manuais/UBEEMAX_REV11.pdf

7. MAXIM INTEGRATED. (2007). MAX3222/MAX3232/MAX3237/MAX3241. Acesso em 30 de Julho de 2015, disponível em Folha de dados do Max3232: http://pdfserv.maximinte grated.com/en/ds/MAX3222-MAX3241.pdf

8. MESSIAS, A. R. (2008). Controle Remoto e Aquisição de Dados via XBee/ZigBee (IEEE 802.15.4) - Acesso em 03 de Julho de 2015, disponível em http://www.rogercom.com /ZigBee/ZigBee.htm

9. PEREZ, F., \& HELENA, E. S. (2011). Estudo de Viabilidade de uso de Redes Sem Fio no Monitoramento de Parques Eólicos. ULBRA. Canoas, RS: ULBRA.

10. RAMOS, J. d. (2012). Instrumentação Eletrônica Sem Fio. São Paulo: Érica.

11. TEXAS INSTRUMENTS. (Outubro de 2014). LM317 3-Terminal Adjustable Regulator. Acesso em 03 de Agosto de 2015, disponível em Folha de dados do LM-317: http://www.ti.com/lit /ds/symlink/Im317.pdf 